\title{
Test characteristics and potential impact of the urine LAM lateral flow assay in HIV-infected outpatients under investigation for TB and able to self-expectorate sputum for diagnostic testing
}

Jonny Peter ${ }^{1,2,7 \dagger}$, Grant Theron ${ }^{1 \dagger}$, Duncan Chanda ${ }^{1,3 \dagger}$, Petra Clowes ${ }^{4,5}$, Andrea Rachow ${ }^{5,6}$, Maia Lesosky ${ }^{7}$, Michael Hoelscher ${ }^{5,6}$, Peter Mwaba ${ }^{3}$, Alex Pym ${ }^{8}$, Keertan Dheda ${ }^{1,9^{*}}$ and For the TB-NEAT team

\begin{abstract}
Background: The commercially available urine LAM strip test, a point-of-care tuberculosis (TB) assay, requires evaluation in a primary care setting where it is most needed. There is currently inadequate data to guide implementation in TB and HIV-endemic settings.
\end{abstract}

Methods: Adult HIV-infected outpatients with suspected pulmonary TB able to self-expectorate sputum from four primary clinics in South Africa, Zambia and Tanzania underwent diagnostic evaluation [sputum smear microscopy, Xpert-MTB/RIF, and culture (reference standard)] as part of a prospective parent study. Urine LAM testing (grade-2 cut-point) was performed on archived samples. Performance characteristics of LAM alone or in combination with sputum - based diagnostics were evaluated. Potential impact on 2 and 6-month morbidity (TBscore), patient dropout rates, and prognosis (death/ loss to follow-up) were evaluated.

Results: Among 583 participants with suspected TB that were HIV-infected or refused testing, the overall LAM sensitivity (95\% Cl; n/N) and in the CD4 $\leq 100$ cells $/ \mathrm{mm}^{3}$ sub-group was $22.7 \%(16.6-28.7 ; 41 / 181)$ and $30.4 \%$ (17.1-43.7; 14/46), respectively. Overall specificity was $93.0 \%$ (90.5-95.6; 361/388). Amongst culture-positive TB cases, adjunctive LAM testing did not improve the sensitivity of either sputum Xpert-MTB/RIF [78.2 \% (69.8-86.7; 72/92) versus $76.1 \%$ (67.4-84.8; 70/92), $p=0.7$ ] or smear-microscopy [56.2 \% (45.9-66.5; 50/89) versus $43.8 \%$ (33.5-54.1; 39/ 89), $p=0.1$ ). Clinic-based LAM, as an adjunct to either smear microscopy or Xpert MTB/RIF same-day testing, would neither have decreased patient dropout, nor increased same-day treatment initiation in this clinical setting where same-day chest radiography was available. LAM positivity was associated with 6-month lost-to-follow-up/death (AOR 4.4; $p=0.002$ ) but not TBscore (at baseline or change in TBscore 2-months post-treatment) $(p=0.17$ ).

Conclusions: In African HIV-TB co-infected outpatients able to self-expectorate sputum LAM had limited sensitivity even at low CD4 counts, and offered no significant incremental diagnostic yield over Xpert-MTB/RIF or smear microscopy. In primary care clinics with chest radiography and where empiric TB treatment is common, LAM seems unlikely to improve rates of same-day treatment initiation and patient dropout, however, the ability of LAM to identify patients at high risk of death or lost-to-follow-up may offer important prognostic value.

Keywords: Tuberculosis, Diagnosis, LAM lateral flow assay, Patient-important impact outcomes

\footnotetext{
*Correspondence: keertan.dheda@uct.ac.za

${ }^{\dagger}$ Equal contributors

${ }^{1}$ Lung Infection and Immunity Unit, Division of Pulmonology \& UCT Lung

Institute, Department of Medicine, University of Cape Town, Cape Town,

South Africa

${ }^{9}$ Institute of Infectious Diseases and Molecular Medicine, University of Cape

Town, Cape Town, South Africa

Full list of author information is available at the end of the article
}

C Biomed Central (c) 2015 Peter et al. This is an Open Access article distributed under the terms of the Creative Commons Attribution License (http://creativecommons.org/licenses/by/4.0), which permits unrestricted use, distribution, and reproduction in any medium, provided the original work is properly credited. The Creative Commons Public Domain Dedication waiver (http:// creativecommons.org/publicdomain/zero/1.0/) applies to the data made available in this article, unless otherwise stated. 


\section{Background}

Of the estimated 8.6 million active tuberculosis (TB) cases globally an estimated three million cases remained either undiagnosed or unreported [1]. Thus, tests and technologies that allow rapid, accurate, point-of-care (POC) diagnosis represent an unmet need and are projected to substantially reduce the global TB burden [2, 3]. An increasing number of high TB/HIV burden settings are implementing frontline Xpert MTB/RIF testing for HIV-infected patients with suspected TB [4-6], although sputum smear microscopy remains the frontline TB diagnostic tool in the majority of resource-poor high burden settings. Used at the POC, Xpert MTB/RIF can decrease dropout rates (patients TB-positive but not returning to initiate treatment) and time-to-treatment initiation [5, 7]. However, sensitivity in sputum is reduced in HIV-infected patients [8] and is also suboptimal in induced sputum samples [9]. These and other considerations including infrastructure and electricity requirements means that countries remain interested in simple low-cost non-sputum based POC diagnostic tools for both pulmonary and extrapulmonary TB diagnosis.

In 2013, the Alere Determine ${ }^{\mathrm{TM}}$ TB LAM Ag lateral flow strip test (Alere, USA, www.alerehiv.com; referred to as LAM from this point forward) became the first commercially available bedside urine test for TB diagnosis in HIV co-infected patients with results available within 25 minutes using just 60ul of urine [10]. To date, diagnostic accuracy studies of urine LAM have been largely single centre [11-13]. Tested patient populations have been heterogeneous (patients with extrapulmonary $\mathrm{TB}$, those unable to spontaneously provide a sputum sample for TB diagnostic testing, or hospitalised patients) [14] and the incremental value of LAM over sputum smear microscopy or Xpert MTB/RIF has thus been variable [11-17]. Nevertheless, POC tests like urine LAM require evaluation in a primary care settings where they are likely to have most impact and where more than $90 \%$ of the TB case load is first encountered. However, there are limited data to guide implementation in such settings where POC TB tests are most needed. Furthermore, the incremental value of LAM over tests like Xpert MTB/RIF, if any, has hardly been studied in HIV endemic primary care settings. There are also limited published data about the potential impact of LAM on morbidity, same-day treatment initiation, patient dropout rates and prognosis $[18,19]$.

In this study we sought to provide multicentre comparative accuracy and extrapolate impact data of adjunctive LAM testing in the setting where the overwhelming majority of individuals with presumptive pulmonary TB present (out-patient primary care clinics). These data are needed to make a definitive recommendation about the use of LAM in this key patient population. We hypothesised that in out-patients able to self-expectorate sputum for sputum-based diagnostics, LAM would have very limited incremental utility. We therefore tested the urine for LAM positivity in a cohort of $583 \mathrm{HIV}$-infected patients with suspected pulmonary TB who formed part of a parent randomised controlled trial comparing Xpert MTB/RIF with same-day smear microscopy in primary care clinics of three sub-Saharan African countries [5]. All the participants provided two expectorated sputa and urine sample.

\section{Methods}

\section{Design and study population}

This cross-sectional accuracy study was nested within a randomised, parallel-arm, multicentre trial, to evaluate the impact of point-of-treatment Xpert MTB/RIF testing with same-day smear microscopy. Patients were enrolled between 12 April 2011 and 1 October 2012. Outpatients $\geq 18$ years that presented to periurban primary-care TB clinics with attached DOTS facilities and microscopy laboratories in Cape Town and Durban (South Africa; laboratory centrally-located), Harare (Zimbabwe), Lusaka (Zambia), and Mbeya (Tanzania) were consecutively enrolled after informed consent. Five Human Research Ethics Committees (HREC) approved the study (University of Cape Town HREC, University of Zambia Biomedical Research Ethics Committee, Mbeya Medical Research Ethics Committee and Medical Research Coordinating Committee of the National Institute for Medical Research, Medical Research Council Durban HREC). Inclusion criteria included: i) symptom(s) of pulmonary TB according to predefined WHO criteria [20, 21] (see online supplementary methods for further details), ii) the ability to spontaneously expectorate two spot sputum specimens with a volume of $\geq 1 \mathrm{ml}$ each, and exclusion criteria included: i) failure to obtain informed consent and ii) initiation of anti-TB treatment in the previous 60 days. In this substudy, HIV-uninfected patients were excluded from the analysis. Patients refusing voluntary counselling and testing for HIV (3\%) where considered "positive" and included in the LAM analysis as this would occur in routine clinical practice given the very high ( $>50 \%$ ) incidence of HIV coinfection amongst new TB cases in these endemic countries. A further detailed description of RCT methodology including description of each primary care clinic site is available with the published manuscript of the parent study [5].

\section{Sample collection and processing}

Each patient had at least two spot expectorated sputa collected sequentially at recruitment. Nurses visually inspected expectorated sputum samples and estimated the volume using standards of known volume. Patients randomised to the smear microscopy study arm received two same-day sputum smears for acid-fast bacilli and 
one arbitrarily selected specimen also underwent culture. Patients in the Xpert MTB/RIF arm received nurseperformed clinic-based Xpert MTB/RIF testing and the other specimen was sent for culture. All patients were asked to provide a spontaneously voided urine sample $(10-30 \mathrm{ml})$ into a sterile receptacle. Urine was transferred to the laboratory within 4 hours and frozen at -20 degrees for later batch testing. Urine samples were collected in all parent study sites except Zimbabwe where urine biobanking was not possible.

\section{Clinical management and follow-up}

Patients were enrolled and initially reviewed by research nursing staff. They were offered voluntary testing and counselling for HIV at recruitment, and received a chest radiograph while awaiting their rapid $\mathrm{TB}$ test result. TB-related morbidity was assessed at enrolment and during 2- and 6-month follow-up using the previously validated TBscore [22] (Additional file 1 Table S1 in the online supplement). Patients were referred to the local DOTS programme office at the same clinic for the initiation of anti-TB treatment if any positive result from either smear microscopy, Xpert MTB/RIF, or TB culture result was obtained. Smear-or Xpert MTB/RIF negative patients were referred to clinical staff for review together with their chest radiographs and as part of the routine clinic workflow. The WHO guidelines for the treatment of smear-negative TB [21] are routinely used at each clinic. Doctors who were not part of the study team and routinely visited each facility twice a week initiated the treatment of smear- or Xpert MTB/RIF-negative patients. Follow-up was conducted for all study patients by research staff at 2- and 6-months post randomisation (within a range of 14 days before and after both time points). Patients were considered lost-to-follow up if they were not contactable despite multiple attempts at telephonic contact and a community healthcare worker tracing.

\section{Diagnostic test procedures}

Liquid TB culture (Bactec MGIT; BD Microbiology Systems, USA) was performed in central laboratories on sputum decontaminated using $\mathrm{N}$-acetyl-L-cysteine- $\mathrm{NaOH}$. Front-loaded, same-day smear microscopy was performed on-site by a technician employed by the programme in a laboratory attached to the healthcare facility, except in South Africa where it was performed at a centralised laboratory. Fluorescence smear microscopy was performed on concentrated samples with auramine-O staining at all sites except Mbeya, Tanzania where concentrated samples underwent $\mathrm{ZN}$-staining and light microscopy. Patients were classified as having smear-positive tuberculosis on the basis of at least one scanty smear (1-9 bacilli per 100 fields $[1000 \times$ for light microscopy and $400 \times$ for fluorescence microscopy]).
Trained staff, including clinical and laboratory staff, according to the manufacturer recommendations on unprocessed thawed urine specimens stored at -20 degrees for not longer than 18 months, performed LAM strip tests. Two independent readers, blinded to reference test results and clinical outcomes, graded ( $0-5$ according to the colour band intensity) each strip after 25-35 minutes using the pre-January 2014 manufacturer's reference card (see Additional file 1 Figure S1A in supplement). Where results between the two independent readers were discordant, a third consensus reader graded the LAM strip, and this consensus read was used in the analysis. Further details on the reading of LAM strip tests and the reference cards are provided in the online supplement. LAM strip results were neither provided to the clinicians caring for patients nor were they used for treatment decisions.

\section{Statistical analyses}

The reference standard for the primary analysis of diagnostic accuracy was a single sputum liquid culture for Mycobacterium tuberculosis. An additional analysis is provided in the online supplement with culture-negative clinical-TB cases considered reference standard positive given the acknowledged limitation of a single sputum TB culture to diagnose TB in HIV co-infection. The study was powered (75-100 \% at $95 \%$ confidence interval) to detect differences in diagnostic accuracy between LAM, Xpert MTB/RIF and smear, alone or in combination, for HIV-infected patients. The study was underpowered to detect small differences in diagnostic accuracy measures amongst different CD4 strata. Descriptive statistics were used to characterise the study population. Diagnostic accuracy measures presented include sensitivity, specificity, positive (LR+, PPV) and negative (LR-, NPV) likelihood ratios and predictive values all with $95 \%$ confidence intervals (CI). $X^{2}$ and Fisher's exact test with mid-P correction were used for comparisons between proportions, and the Mann-Whitney test was used to compare differences in TBscore. The potential impact of same-day LAM testing was assessed by assuming that LAM testing would be performed at the first point of clinic contact and all LAMpositive patients would initiate anti-TB treatment immediately. $X^{2}$-squared testing is used to compare same-day treatment initiation and treatment dropout proportions with and without the use of LAM in each study arm. Multivariable- linear (for morbidity scores) and logistic (for mortality) regression analyses were performed. Sample size calculations were based on the primary outcome for the parent study [5] (http://clinicaltrials.gov/show/ NCT01554384). Based on the prevalence of HIV and culture-positive TB cases in the parent study there was adequate study power for the $95 \% \mathrm{CI}$ around LAM accuracy measures to be within a range of $\pm 10 \%$. Analyses were 
performed using OpenEpi (version 2.3.1) [23], and R (version 3.0) [24]. The study is reported in accordance with the STARD initiative recommendations [25].

\section{Role of the funding source}

Alere donated the LAM strip tests. However, neither the company nor the study sponsor had any role in study design, data collection, data analysis, data interpretation, or writing of the report. The corresponding author had full access to all the data in the study and had final responsibility for the decision to submit for publication.

\section{Results}

\section{Study sites and population characteristics}

Figure 1 provides the study profile. We enrolled 1095 patients with suspected TB, that were able to provide $\geq 2$ sputa and a spot urine sample, from four primary care clinics in South Africa $(n=419$ in Cape Town, $n=193$ in Durban), Zambia $(n=400)$ and Tanzania $(n=83)$. Further detailed descriptions of each primary care clinic have already been published with the parent study [5]. HIV-infection was confirmed in 564/1095 (52 \%) and testing was refused in 19/1095 (1\%); LAM test performance is considered in these two groups combined $(\mathrm{N}=583)$. Of the $583 \mathrm{HIV}$-positive/status unknown patients with suspected TB, $14(2 \%)$ had either a contaminated culture or no available result, and 181 (31\%) had culture-positive TB. Table 1 provides the basic demographic and clinical characteristics of the HIV-infected patients of the study population stratified by study site.

\section{LAM performance}

Three ( $<1 \%)$ LAM strip tests failed on the first attempt and required use of a second strip test to produce valid results. The $3^{\text {rd }}$ LAM strip reader was required in 151/ $583(26 \%)$ patients, and in 126/151 (83 \%) cases this was for differences in grading bands between the grade 0 and 1 intensity. Thus, excluding grade $0 / 1$ discordance a $3^{\text {rd }}$ reader was required in only 25/583 (4\%) of patients. Additional file 1 Table S2 compares LAM diagnostic accuracy using the grade- 2 versus grade- 1 cut-point (pre-January 2014 reference card, Fig. 1A) showing the higher specificity and LR+ of the grade- 2 cut-point. Interobserver agreement as to the presence versus absence of a test band of intensity grade 2 or higher was $97.3 \%$ (kappa 0.87), while agreement regarding the presence versus absence of a test band of intensity grade 1 or higher was $90.9 \%$ (kappa 0.77), $\mathrm{p}<0.001$. Based on this data, and previously published work [14-16, 26] and in accordance with the updated manufacturer's reference card (see Additional file 1 Figure S1B), the remaining diagnostic accuracy data presented in the results section is presented using the grade-2 LAM cut-point.

Table 2 shows the diagnostic accuracy of LAM, Xpert MTB/RIF and smear-microscopy amongst HIV-infected patients stratified by CD4 cell count (PPV, NPV, LR+ and LR- shown in Additional file 1 Table S3). Overall, LAM had sensitivity $(95 \% \mathrm{CI})$ and specificity $(95 \% \mathrm{CI})$ of $22.7 \%(16.6-28.7)$ and 93.0 (90.5-95.6) respectively. LAM specificity did not significantly increase when TB culture-negative patients diagnosed with clinical-TB

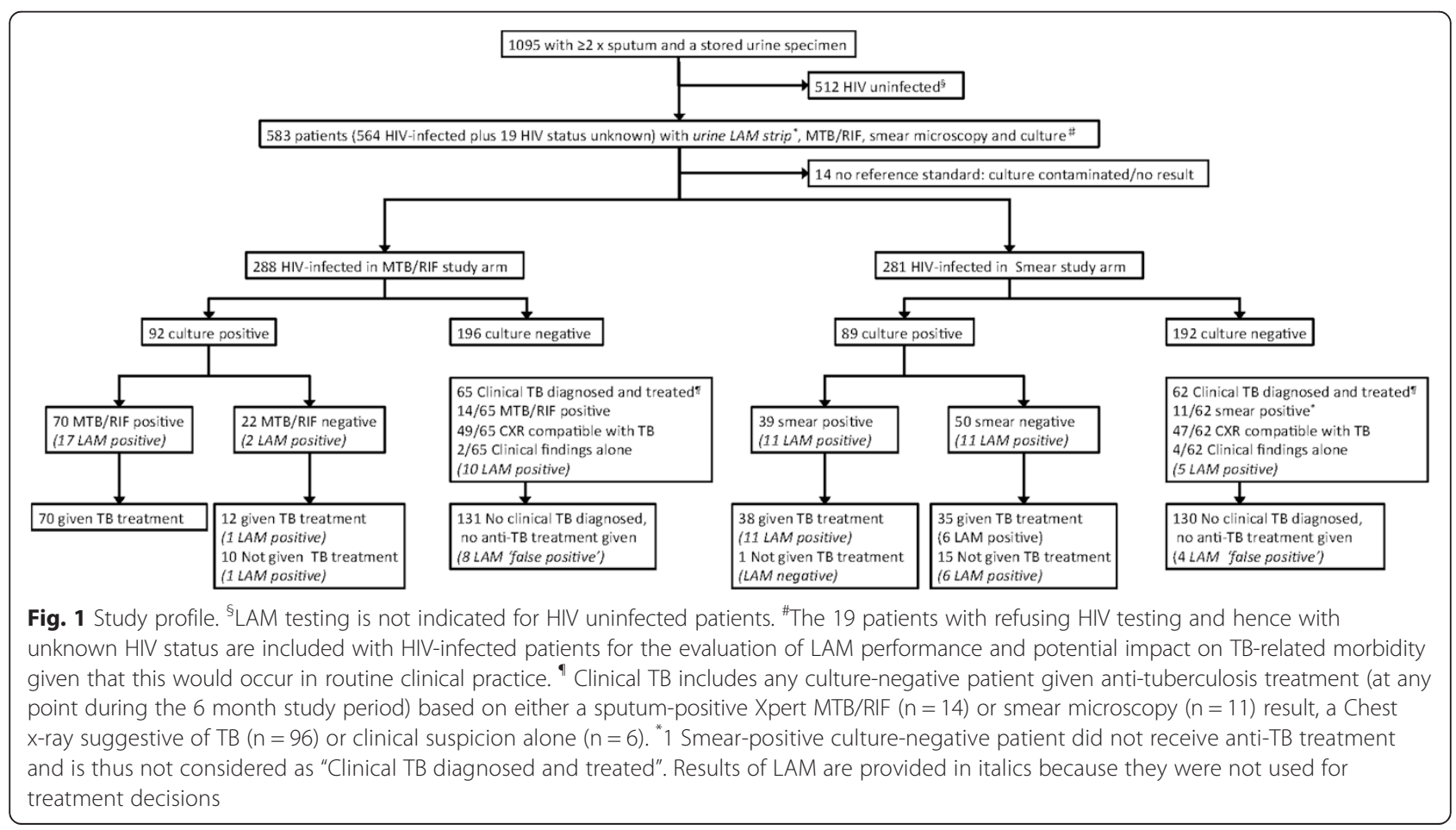


Table 1 Study site and population characteristics

\begin{tabular}{|c|c|c|c|c|c|}
\hline & $\begin{array}{l}\text { Gugulethu TB Clinic } \\
\text { (Cape Town, South Africa) }\end{array}$ & $\begin{array}{l}\text { Kanyama TB Clinic } \\
\text { (Lusaka, Zambia) }\end{array}$ & $\begin{array}{l}\text { St. Mary's Day Clinic } \\
\text { (Durban, South Africa) }\end{array}$ & $\begin{array}{l}\text { Ifisi Day Clinic } \\
\text { (Mbeya, Tanzania) }\end{array}$ & Overall \\
\hline No. patients enrolled in parent RCT & 419 & 400 & 193 & 83 & 1095 \\
\hline HIV-infected & $133(32)$ & $268(67)$ & $114(59)$ & $49(59)$ & $564(52)$ \\
\hline HIV uninfected & $278(66)$ & $130(33)$ & $70(35)$ & $34(41)$ & $512(47)$ \\
\hline HIV testing refused & $8(2)$ & $2(<1)$ & $9(5)$ & $0(0)$ & $19(1)$ \\
\hline \multicolumn{6}{|c|}{ Demographics of HIV-infected/testing refused patients included in primary analysis $(N=583)$} \\
\hline & $N=141$ & $N=270$ & $N=123$ & $N=49$ & $N=583$ \\
\hline Median age, years (range, IQR) & $35(19-79,30-40)$ & $35(19-79,30-40)$ & $36(20-63,29-42)$ & $36(17-72,33-48)$ & $36(17-79,30-41)$ \\
\hline Women $(\%)^{a}$ & $81(58)$ & $99(37)$ & $65(53)$ & $27(55)$ & $272(46)$ \\
\hline Previous TB ${ }^{\mathrm{b}}(\%)$ & $69(49)$ & $42(16)$ & $37(30)$ & $2(4)$ & $150(26)$ \\
\hline $\begin{array}{l}\text { Median CD4 cell count, cells/ml } \\
(\text { range, IQR) }\end{array}$ & $261(6-1089,137-410)$ & $201(1-1251,104-339)$ & $231(2-1157,106-494)$ & $91(1-423,33-213)$ & $210(1-1251,103-375)$ \\
\hline HIV-infected on ART ${ }^{d}(\%)$ & $51 / 133(38)$ & $54 / 268(20)$ & 28/114 (25) & 2/49 (4) & 135/564 (24) \\
\hline \multicolumn{6}{|l|}{ TB outcomes } \\
\hline $\begin{array}{l}\text { Sputum TB culture-positive } \\
\text { patients }^{\mathrm{e}}(\%)\end{array}$ & $39(28)$ & $100(37)$ & $25(20)$ & $17(35)$ & $181(31)$ \\
\hline Clinical TB ${ }^{f}(\%)$ & $17(12)$ & $26(10)$ & $6(5)$ & $4(8)$ & $53(9)$ \\
\hline \multicolumn{6}{|c|}{ TB-related morbidity at baseline in culture-positive patients } \\
\hline Median TBscore $(\mathrm{IQR})^{\mathrm{g}}$ & $4(3-5)$ & $6(4-7)$ & $5(4-6)$ & $7(5-9)$ & $5(4-7)$ \\
\hline
\end{tabular}

Abbreviations: $I Q R$, interquartile range; $T B$, tuberculosis

Footnotes: ${ }^{\mathrm{a}} \mathrm{A}$ smaller proportion of patients in Lusaka were female compared to Cape Town, Durban and Mbeya ( $\mathrm{p}$-values of $<0.0001,0.003$, and 0.015 )

${ }^{\mathrm{b}} \mathrm{A}$ greater proportion of patients in Cape Town had a history of previous TB compared to Lusaka, Durban, and Mbeya ( $p$-values of $<0.0001,0.002$, and $<0.0001$ )

${ }^{c} H I V$-infected patients in Mbeya had lower CD4 cell counts compared with Cape Town, Lusaka and Durban ( $p$-value of $<0.001$ for all comparisons)

${ }^{\mathrm{d}} \mathrm{A}$ greater proportion of HIV-infected patients were on ART in Cape Town compared to Lusaka, Durban, and Mbeya (p-value of $<0.0001$ for all comparisons)

${ }^{\mathrm{e}} \mathrm{A}$ lower proportion of patients in Durban were culture-positive for TB compared to Lusaka and Mbeya ( $p$-values $<0.001$ and 0.05 )

${ }^{f}$ The median TBscore in patients from Mbeya was higher than Cape Town, Lusaka or Durban ( $p$-values $<0.0001$ for all comparisons)

${ }^{g} \mathrm{~A}$ greater proportion of patients culture-negative patients received TB treatment empirically in Cape Town compared to Durban ( $p=0.039$ )

were considered reference test positive in a secondary analysis [95.4\% (92.1-97.4) versus 93.0 (90.5-95.6), $\mathrm{p}=$ 0.2 , Additional file 1 Table S4]. LAM specificity was significantly higher at South African compared to Zambian and Tanzanian study sites, both in the primary and secondary analyses [Primary analysis: Cape Town: $99.0 \%$ and Durban: 97.9 \% compared to Lusaka: $87.1 \%$ and Mbeya: $89.7 \%, \mathrm{p}<0.001$ for comparisons between SA sites and Lusaka and $\mathrm{p}<0.05$ for comparisons between SA sites and Mbeya] (see online Additional file 1 Table S5B and C)]. LAM strip sensitivity $(95 \% \mathrm{CI})$ was not significantly higher in CD $4 \leq 100$ versus $>100$ cell $/ \mathrm{mm}^{3}$ [30.4\% (17.1-43.7) versus $18.3 \%(12.5-25.9), \mathrm{p}=0.085$ ], while specificity was similar.

\section{LAM testing compared to Xpert MTB/RIF or smear microscopy}

The overall sensitivity (95\% CI) of Xpert MTB/RIF $(76.1 \%, 70 / 92)$ was significantly higher than either LAM $(22.7 \%, 41 / 181)$ or smear microscopy $(43.8 \%, 39 / 89)$ ( $\mathrm{p}<0.001$ for both comparisons); similarly in patients with CD4 $\leq 100$ cells $/ \mathrm{mm}^{3}$ Xpert MTB/RIF (76.2 \%, 16/21) offered higher sensitivity than either LAM $(30.4 \%, 14 / 46$, $\mathrm{p}<0.001)$ or smear microscopy (36 \%, 9/25, $\mathrm{p}=0.006)$.
Xpert MTB/RIF had the highest test LR+ $(95 \% \mathrm{CI})$ of 10.7 (9.2-12.4) and correspondingly a PPV (95 \% CI) of $83.3 \%$ (74.0-89.8). Overall, but not in patients with $\mathrm{CD} 4 \leq 100$ cells $/ \mathrm{mm}^{3}$, smear microscopy $(43.8 \%, 39 / 89)$ offered significantly higher sensitivity compared to LAM alone $(22.7 \%, 41 / 181, \mathrm{p}<0.001)$. Similar differences in test sensitivities were seen across all study sites except in Durban where LAM (40 \%), sputum Xpert MTB/RIF (60\%) and smear microscopy $(50 \%)$ offered similar sensitivities (online Additional file 1 Table S5A). Specificity of LAM (93.0 \%), Xpert MTB/RIF (92.9 \%) and smear microscopy (93.2 \%) used alone was similar irrespective of CD4 strata ( $>0.05$ for all comparisons).

\section{LAM in combination with either sputum-based MTB/RIF or smear microscopy}

The diagnostic accuracy of using LAM in combination with either sputum-based Xpert MTB/RIF or smearmicroscopy is also shown in Table 2. LAM plus Xpert MTB/RIF (72/92, $78.3 \%$ ) did not offer significantly better sensitivity to Xpert MTB/RIF alone (70/92, $76.1 \%)(\mathrm{p}=0.7)$. Similarly, LAM plus smear microscopy $(50 / 89,56.2 \%)$ did not offer significantly better sensitivity to smear microscopy alone $(39 / 89,43.8 \%)(p=0.1)$. In contrast, the specificity of 
Table 2 Key diagnostic accuracy measures of LAM (grade 2 cut-point), sputum Xpert MTB/RIF or smear microscopy alone or in combination for culture-confirmed versus culture-negative pulmonary tuberculosis amongst HIV-infected (and refused testing) patients stratified by CD4 cell count

\begin{tabular}{|c|c|c|c|c|}
\hline \multirow[t]{2}{*}{ Diagnostic (s) } & \multirow[b]{2}{*}{$\mathrm{n} / \mathrm{N}$} & \multicolumn{2}{|l|}{ Sensitivity } & \multirow{2}{*}{$\begin{array}{l}\text { Specificity } \\
\%(95 \% \text { Cl) }\end{array}$} \\
\hline & & $\%(95 \% \mathrm{Cl})$ & $\mathrm{n} / \mathrm{N}$ & \\
\hline \multicolumn{5}{|l|}{ LAM alone $^{a}$} \\
\hline HIV-infected & $41 / 181$ & $22.7(16.6-28.7)$ & $361 / 388$ & $93.0(90.5-95.6)$ \\
\hline CD4 $\leq 100$ cells $/ \mathrm{mm}^{3} \mathrm{~b}$ & $14 / 46$ & $30.4(17.1-43.7)$ & $70 / 75$ & $93.3(87.7-99.0)$ \\
\hline CD4 > 100 cells $/ \mathrm{mm}^{3}$ & $23 / 126$ & $18.3(12.5-25.9)$ & $257 / 274$ & $93.8(90.3-96.1)$ \\
\hline \multicolumn{5}{|l|}{ Xpert MTB/RIF alone } \\
\hline HIV-infected & $70 / 92$ & $76.1(67.4-84.8)$ & 182/196 & $92.9(88.4-95.7)$ \\
\hline $\mathrm{CD} 4 \leq 100 \mathrm{cell} / \mathrm{s} / \mathrm{mm}^{3}$ & $16 / 21$ & $76.2(58.0-94.4)$ & $36 / 40$ & $90.0(80.7-99.3)$ \\
\hline CD4 > 100 cells $/ \mathrm{mm}^{3}$ & $47 / 63$ & $74.6(62.7-83.7)$ & $130 / 138$ & $94.2(89.0-97.0)$ \\
\hline \multicolumn{5}{|l|}{ Smear alone } \\
\hline HIV-infected & $39 / 89$ & $43.8(34.0-54.2)$ & $179 / 192$ & $93.2(88.8-96.0)$ \\
\hline CD4 $\leq 100$ cells $/ \mathrm{mm}^{3}$ & $9 / 25$ & $36.0(17.2-54.8)$ & $31 / 35$ & $88.9(78.6-99.1)$ \\
\hline CD4 > 100 cells $/ \mathrm{mm}^{3}$ & $30 / 63$ & $47.6(35.8-59.7)$ & $129 / 136$ & $94.9(89.8-97.5)$ \\
\hline \multicolumn{5}{|c|}{ Xpert MTB/RIF and LAM combined ${ }^{c}$} \\
\hline HIV-infected & $72 / 92$ & $78.3(69.8-86.7)$ & 169/196 & $86.2(81.4-91.0)$ \\
\hline $\mathrm{CD} 4 \leq 100 \mathrm{cell} / \mathrm{s} / \mathrm{mm}^{3}$ & $18 / 21$ & $85.7(70.7-100.1)$ & $35 / 40$ & $87.5(77.3-97.7)$ \\
\hline CD4 > $100 \mathrm{cells} / \mathrm{mm}^{3}$ & $47 / 63$ & $74.6(62.7-83.7)$ & $121 / 138$ & $87.7(81.2-92.2)$ \\
\hline \multicolumn{5}{|c|}{ Smear and LAM combined ${ }^{c}$} \\
\hline HIV-infected & $50 / 89$ & $56.2(45.9-66.5)$ & 172/192 & $89.6(85.3-93.9)$ \\
\hline $\mathrm{CD} 4 \leq 100 \mathrm{cell} / \mathrm{s} / \mathrm{mm}^{3}$ & $11 / 25$ & $44.0(24.5-63.5)$ & $28 / 35$ & $80.0(66.7-93.3)$ \\
\hline CD4 > 100 cells $/ \mathrm{mm}^{3}$ & $38 / 63$ & $60.3(48.0-71.5)$ & $125 / 136$ & $91.9(86.1-95.4)$ \\
\hline
\end{tabular}

${ }^{\mathrm{a}} 14 / 583 \mathrm{HIV}$-infected and test refused patients had no reference standard result (see Fig. 1) and therefore a total of 569 patients were used for evaluation of urinary LAM performance

$\mathrm{b}_{48 / 569}$ HIV-infected patients with LAM test results missing CD4 cell count data. For no diagnostic accuracy measure did any of the diagnostic tests, either alone or in combination, performed significantly better in CD4 $\leq 100$ cells $/ \mathrm{mm}^{3}$ compared to CD4 $>100$ cells $/ \mathrm{mm}^{3}(p>0.05)$

'Either test positive is considered as a "positive" result

LAM plus Xpert MTB/RIF (169/196, $86.2 \%$ ) was significantly lower than either LAM strip (361/388, $93.0 \%$ ) or Xpert MTB/RIF (182/189, $92.9 \%)$ testing alone $(\mathrm{p}=0.007$ versus $L A M$ and $p=0.032$ versus Xpert $M T B / R I F)$, and these relationships were unchanged if the analysis was restricted to patients with CD $4 \leq 100$ cells $/ \mathrm{mm}^{3}$. For LAM plus smear microscopy, the overall specificity $(172 / 192$, $89.6 \%)$ did not differ significantly compared to either LAM (361/388, $93.0 \%)$ or smear (179/192, $93.2 \%)$ alone ( $p>0.05$ for both comparisons), however, in patients with CD4 $\leq$ 100 cells $/ \mathrm{mm}^{3}$ combined specificity was lower than LAM testing alone $(93.0 \%)(\mathrm{p}=0.037)$. The specificity relationships were unchanged in the secondary analysis (Additional file 1 Table S4).

A sensitivity analysis was performed to investigate the impact of the $21 \%(28 / 131)$ and $27 \%(36 / 130)$ Non-TB patients in the smear and Xpert study arms respectively that were lost-to-follow up and the $1.5 \%(2 / 131)$ and $3 \%$ $(4 / 130)$ that were deceased. No differences in the diagnostic accuracy of LAM alone or with smear/Xpert were noted (data not shown).
Potential impact on patient-important treatment outcomes of adding point-of-care LAM to sputum-based Xpert MTB/RIF or smear microscopy

In the parent study, the reason for treatment initiation, time-to-treatment and failure to initiate treatment in culture-positive TB patients ('dropout') was recorded (Fig. 2). The potential impact on these outcomes of adding point-of-care LAM (in the manner described in methods section) is also shown in Fig. 2. In the smear microscopy study arm with the use of empiric treatment based on CXR, use of POC LAM would neither have significantly decreased dropout [18\%(16/89) to $12 \%$ (11/89), $\mathrm{p}=0.3]$, nor significantly increased same-day treatment initiation [35\%(31/89) to $44 \%(39 / 89), \mathrm{p}=$ $0.2]$. However, in primary care settings without same-day CXR facilities, POC LAM would have significantly increased same-day treatment initiation [24\% (21/89) to $44 \%(39 / 89, \mathrm{p}=0.004]$. Potentially unnecessary treatment initiation would have increased by $3 \%(4 / 116)$ due to LAM 'false-positives'. In the Xpert MTB/RIF study arm, LAM would neither have significantly decreased 


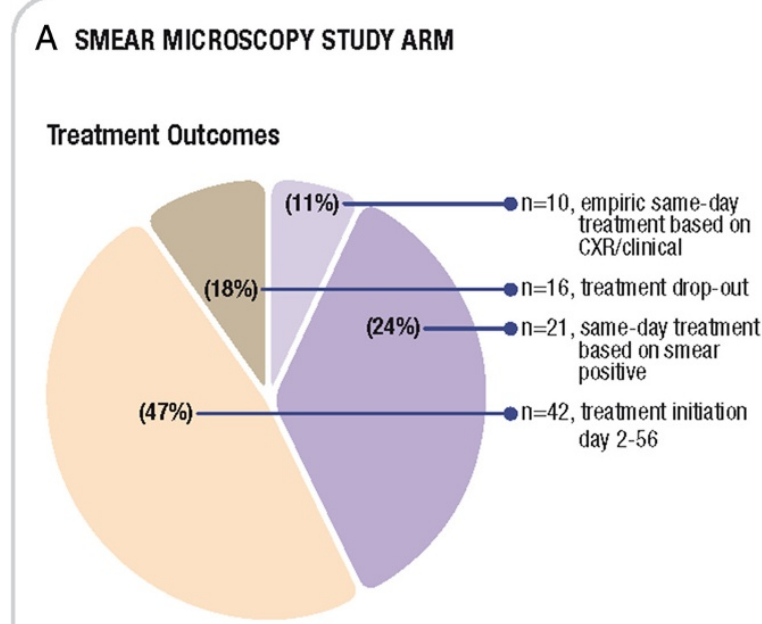

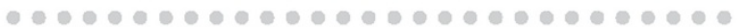

Possible treatment outcomes (with addition of LAM testing)

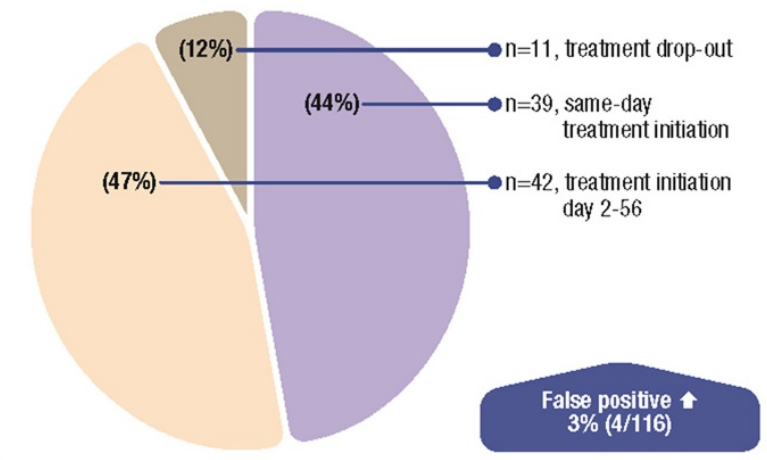

\section{B XPERT MTB/RIF STUDY ARM}

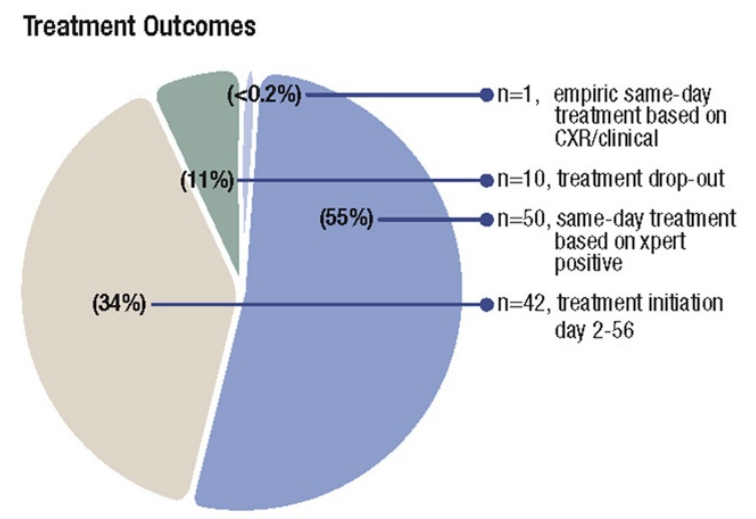

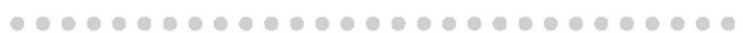

Possible treatment outcomes (with addition of LAM testing)

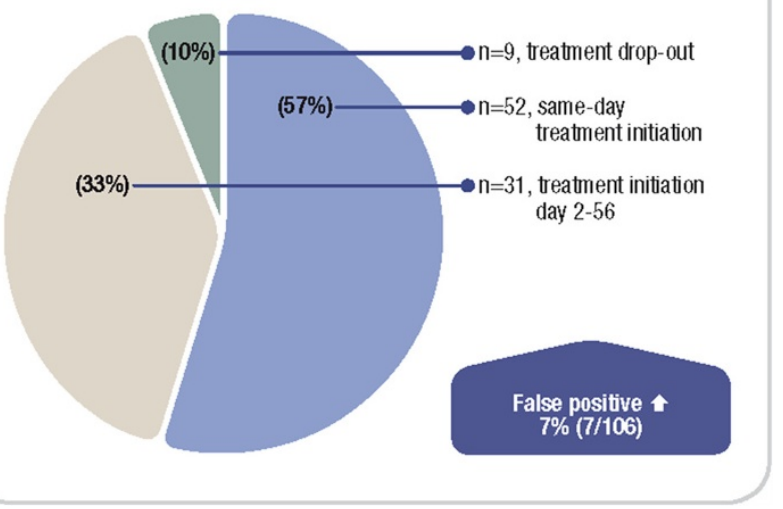

Fig. 2 Treatment outcomes in each study arm indicating the potential impact of LAM in TB culture positive patients. a Smear-microscopy b clinic-based Xpert MTB/RIF testing. 'False-positives': These indicate patients that were sputum culture-negative, smear or Xpert MTB/RIF-negative and not given empiric TB treatment by the attending clinician that would have been LAM positive. These patients would have thus received inappropriate anti-TB treatment

treatment dropout [11 \% (10/92) to $10 \%(9 / 92), \mathrm{p}=0.8]$ nor significantly increased same-day treatment initiation [55 \% (51/92) to $57 \%$ (52/92), p = 0.9]. However, potentially unnecessary treatment initiation would have increased by 7 \% (7/106) due to LAM 'false-positives'.

\section{LAM as a potential prognostic marker}

Table 3 shows all cause 6-month mortality, lost-to-followup and TBscore at enrolment amongst HIV-infected/status unknown patients stratified by LAM result. LAM strip positive versus negative patients had: i) higher all cause 6-month mortality [25\% versus $11 \%, \mathrm{P}=0.02$ ], ii) higher lost-to-follow-up [54 \% versus $30 \%, \mathrm{p}<0.001$ ], and iii) a higher baseline TB-related morbidity score [7 (6-8) versus 5 (4-7), $\mathrm{p}=0.03$ ]. However, in the multivariate analyses shown in Table 4, LAM positivity was only a predictor of the combined outcome of lost-to-follow-up/death but not 6-month mortality or baseline TBscore. In addition, TB-related morbidity outcomes, as measured by the amount of improvement from enrolment in TB-score at 2- and 6-months post-initiation of effective anti-TB treatment were similar amongst LAM strip positive versus negative patients [ $4(3-5)$ versus $4(2.5-5), \mathrm{p}=0.136$ ] (online Additional file 1 Table S6).

\section{Discussion}

In this multi-centre out-patient study of patients with suspected PTB able to provide expectorated sputum for diagnostic testing, LAM had poor overall sensitivity, which did not significantly improve in patients with CD4 cell counts less than 100 cells/ml. LAM was not able to improve on the diagnosis offered by either sputum-based Xpert MTB/RIF or smear microscopy alone. Due to this lack of incremental utility over sputum-based 
Table 3 Morbidity (measured by TBscore), 6-month all cause mortality and lost-to-follow up stratified by baseline results of LAM in HIV-infected (or test refused) patients ${ }^{a}$

\begin{tabular}{lllll}
\hline & All HIV-infected $\mathrm{n} / \mathrm{N}(\%)$ & LAM strip positive $\mathrm{n} / \mathrm{N}(\%)$ & LAM strip negative n/N (\%) & P-value \\
\hline 6-month mortality $(\mathrm{N}=393)^{\mathbf{b}}$ & $49 / 393(13)$ & $9 / 32(25)$ & $40 / 361(11)$ & 0.021 \\
TB culture-positive only $(\mathrm{N}=123)^{\mathrm{b}}$ & & $6 / 17(35)$ & $15 / 106(14)$ & $152(30)$ \\
Lost-to-follow up & $190 / 583(33)$ & $38(54)$ & $34 / 140(24)$ & \\
Culture-positive $(\mathrm{N}=58)$ & & $24 / 41(59)$ & & $<032$ \\
TBscore at enrolment & & & $5(4-7)$ & $<0.001$ \\
Culture-positive $(\mathrm{N}=181)$ & $6(4-7.5)$ & $7(6-8)$ & 0.025 \\
\hline
\end{tabular}

${ }^{a} 14$ HIV-infected and test refused patients with no reference standard result (see Fig. 1) excluded from the analyses of index test diagnostic accuracy are included in this analysis of morbidity and mortality. Thus a total of 583 patients are included in this table's analysis

${ }^{\mathrm{b}}$ Of the 583 HIV-infected patients with a LAM result, 190 were lost-to-follow-up at 6-months. Of the 393 patients with complete 6-month follow-up data, 123 were culture-positive TB

tools, LAM seems unlikely to be able to improve outpatient important treatment outcomes. Indeed, although LAM could offer some prognostic utility in identifying patients at high risk of death or loss-to-follow-up, there was no impact on patient dropout or morbidity. Adjunctive LAM testing may only increase same-day treatment initiation in clinic settings using sputum smear microscopy where same-day chest radiography is not available to guide empiric sameday treatment decisions. It may be argued that our study only considered outpatients able to expectorate sputum, and LAM may offer important incremental value for patients unable to provide sputum for diagnostic testing. However, this study's patient population is the major subgroup presenting to primary care facilities for frontline testing and although preliminary data suggest that this is not the subgroup most likely to benefit from LAM, data is nevertheless essential for clear recommendations to guide implementation across TB programs. Indeed, program directors continue to question why the only commercially available and affordable POC TB test is unavailable for frontline testing? The data outlined here offers valuable insights.

Published data, albeit limited, indicate that LAM sensitivity is increased with higher circulating LAM levels, occurring with higher mycobacterial disease burden, extrapulmonary TB, lower CD4 cell count and WHO clinical stage 3 and 4 in out- and in-patient settings [11, 27-34]. Moreover, LAM and sputum smear microscopy identified non-overlapping sub-groups of culture-positive $\mathrm{TB}$, thereby offering additive diagnostic value $[11-14,16$, 35]. By contrast, we found no incremental benefit of LAM. There are a number of possible explanations. Firstly, sputum-scarce TB, smear-negative TB, and EPTB is more common in hospital-based and pre-ART screening cohorts and thus smear microscopy sensitivity is more likely to be reduced, which in turn would increase the incremental benefit offered by LAM testing. Secondly, differences in sputum smear microscopy staining and concentration methodologies across different studies affect smear microscopy sensitivity [13]. Thirdly, in contrast to other LAM-based studies [11, 12, 14] our study did not offer sputum induction to improve sputum sampling and thus, those unable to spontaneously produce two spot sputa were excluded. Sputum induction was not offered as the pragmatic study design of the parent study reflects the reality that sputum induction facilities remain unavailable in the majority of routine primary care clinic settings. Nevertheless, the inability of LAM testing to improve the diagnostic yield of a single sputum-based Xpert MTB/RIF, irrespective of declining CD4 cell count, is consistent with the findings of Lawn et al. and reflect the superior sensitivity of sputum-based Xpert MTB/RIF for the diagnosis of pulmonary TB [11, 36, 37].

In our initial study of LAM amongst hospitalised HIVinfected patients with advanced immunosuppression we noted that test specificity and inter-reader agreement was optimised ( $>95 \%$ for both) by use of an alternative grade- 2 rather than the manufacturer's initially suggested grade-1 cut-point [16]. Based on these findings, the manufacturer's reference card has been updated as of January 2014 so that the first positive visual band corresponds to the grade- 2 intensity band of the old reference card (see Fig. 1 of the online supplement, www.alere.com).

Independent of cut-point selection, suboptimal LAM specificity ( $<95 \%$ as recommended by an expert committee for point-of-care TB testing [38]), particularly in countries north of South Africa, remains a concern. Indeed we noted lower test specificity in Tanzania and Zambia compared to the two South African study sites. Reasons for this may include different degrees of the unavoidable misclassification bias associated with a single sputum culture to correctly classify TB in HIV-infected patients with advancing immunosuppression. However, in our secondary analysis excluding 'probable or clinical TB' the specificity in Zambia and Mbeya remained lower. Kroidl et al. found cross-contamination of the LAM ELISA from dust, soil and stool in Tanzania, and we have demonstrated cross-reacting LAM-like glycolipid antigens in Nocardia and Candida spp. [30, 39]. Sterile collection of urine samples is essential, especially 
Table 4 Multivariate analysis showing predictors of all-cause mortality, lost-to-follow-up/mortality and baseline TB-related morbidity in HIV-infected patients overall and restricted to culture-positive TB patients only ${ }^{a}$

\begin{tabular}{|c|c|c|c|c|}
\hline & $P$-value & $\mathrm{AOR}$ & Lower $95 \% \mathrm{Cl}$ & Upper $95 \% \mathrm{Cl}$ \\
\hline \multicolumn{5}{|l|}{ 6-month all-cause mortality } \\
\hline \multicolumn{5}{|l|}{ All HIV-infected $\left(n^{*}=173\right)$} \\
\hline Age, years & 0.051 & 0.934 & 0.867 & 0.997 \\
\hline CD4 cell count, cells $/ \mathrm{mm}^{3}$ & 0.038 & 0.996 & 0.991 & 0.999 \\
\hline TB culture positive (pos/neg) & 0.007 & 9.207 & 2.069 & 53.4 \\
\hline Baseline TB score & 0.050 & 1.349 & 1.012 & 1.86 \\
\hline LAM strip result ${ }^{b}$ & 0.170 & 2.762 & 0.613 & 11.72 \\
\hline \multicolumn{5}{|l|}{ TB culture positive $(n=117)$} \\
\hline CD4 cell count, cells $/ \mathrm{mm}^{3}$ & 0.069 & 0.9951 & 0.989 & 0.999 \\
\hline Baseline TB score & 0.007 & 1.683 & 1.19 & 2.555 \\
\hline LAM strip result & 0.252 & 2.59 & 0.495 & 13.72 \\
\hline \multicolumn{5}{|l|}{ Lost-to-follow-up or deceased } \\
\hline \multicolumn{5}{|l|}{ All HIV-infected $(n=268)$} \\
\hline Age, years & 0.048 & 0.966 & 0.932 & 0.999 \\
\hline Zambia & $<0.0001$ & 12.52 & 4.077 & 45.15 \\
\hline Durban, South Africa & 0.052 & 3.701 & 1.027 & 14.82 \\
\hline CD4 cell count, cells $/ \mathrm{mm}^{3}$ & 0.058 & 0.998 & 0.997 & 1 \\
\hline LAM strip result & 0.002 & 4.382 & 1.834 & 11.58 \\
\hline \multicolumn{5}{|l|}{ TB culture positive $(n=170)$} \\
\hline Age, years & 0.010 & 0.944 & 0.903 & 0.985 \\
\hline Zambia & 0.002 & 5.897 & 2.055 & 18.61 \\
\hline Baseline TB score & 0.006 & 1.301 & 1.085 & 1.583 \\
\hline LAM strip result & 0.007 & 4.717 & 1.638 & 15.85 \\
\hline \multicolumn{5}{|l|}{ Baseline TB score $(n=146)$} \\
\hline \multicolumn{5}{|l|}{ TB culture positive } \\
\hline CD4 cell count, cells $/ \mathrm{mm}^{3}$ & 0.02716 & 0.9977 & 0.9958 & 0.9997 \\
\hline LAM strip result (pos/neg) & 0.1771 & 1.886 & 0.7542 & 4.717 \\
\hline
\end{tabular}

Abbreviations: $A R V$, anti-retroviral; $C l$, confidence interval; $A O R$, adjusted odds ratio

${ }^{a}$ Additional co-variates included all multivariate models as potential confounders include: study arm (Xpert MTB/RIF or smear), study site, sex, age. Additional co-variates evaluated as additional predictors of outcomes included: positive initial TB result (Xpert MTB/RIF or smear), ARV treatment status, previous TB and CXR suggestive of TB. LAM strip test is always shown while only significant co-variates in each multivariate regression are shown

${ }^{*} \mathrm{n}=$ number included in each of the multivariate models depending on outcome and sub-group considered

${ }^{b}$ LAM strip results are considered as a binary variable (positive/negative) using the grade 2 cut-point. Results of the multivariate analysis do not change if LAM strip results are considered as continuous variable from grade $0-5$

in countries north of South Africa, and in a recent Ugandan in- and out-patient study LAM specificity was $95 \%[14]$.

LAM offered limited incremental diagnostic benefit over sputum-based diagnostics. In contrast to studies showing incremental benefit of LAM in hospitalised patients with sputum-scarce TB and EPTB $[14,16]$ or those identifying patients with $\mathrm{TB}$ missed by empiric treatment initiation but identified by LAM [40]. Consequently, our study suggests LAM would have minimal potential impact on patient important treatment outcomes. In fact, test specificity was significantly lower when combining Xpert MTB/RIF with LAM for both a culture and composite reference standard with the potential to increase inappropriate treatment initiation. Thus, sputum-based diagnosis, especially where Xpert MTB/RIF is available, should be preferred in HIVinfected outpatients able to spontaneously provide sputa. However, LAM may potentially improve same-day treatment initiation in the clinic setting where only sputum smear microscopy is performed and no chest radiography facilities are available. In addition, LAM may still offer i) important added diagnostic benefit where the performance of sputum-based tests is reduced such as 
sputum-scarce TB, extrapulmonary TB, mycobacteremia [27], and/ or renal TB [41], and ii) important prognostic and treatment monitoring utility [18].

This study had several limitations and strengths. It is the first large multicentre study in primary care practice, allowing for accurate evaluation of diagnostic accuracy across three sub-Saharan African countries. The design of the parent study offered a unique opportunity to evaluate the diagnostic accuracy of LAM in a well-defined outpatient population able to provide sputum for diagnostic testing and hence, to estimate the potential impact of LAM when combined with either Xpert MTB/RIF or smear in this patient group. Misclassification bias was a potential problem in our study as a single sputum culture can miss TB cases amongst HIV-infected patients. However, in an alternative analysis where TB culture-negative patients diagnosed as clinical TB and initiating treatment are considered the reference test, no significant difference in LAM specificity was noted and study conclusions were unaltered. In addition, a sensitivity analysis with lostto-follow-up and deceased patients excluded from the non-TB group i.e. considered unclassifiable, did not significantly alter diagnostic accuracy measures. LAM was performed on frozen rather than fresh samples which could have reduced test sensitivity, however, meta-analysis data suggests no differences in diagnostic accuracy using frozen rather than fresh samples [42]. Consequently, impact data for adjunctive LAM is extrapolated. The study was not powered to detect small differences in sub-groups (CD4 strata, treatment dropouts) and thus small incremental benefits of LAM in these sub-groups may not have been detected. Likewise, the small number of study deaths limited power to examine LAM as a predictor of mortality in the multivariate analysis.

In conclusion, LAM strip testing had poor sensitivity amongst HIV-infected outpatients able to provide expectorated sputum for diagnostic testing. There was no incremental diagnostic benefit over either Xpert MTB/RIF or smear microscopy. If used as an adjunctive diagnostic tool in this setting, it is unlikely to impact patientimportant treatment outcomes such as morbidity, patient dropout, or same-day treatment initiation, except in smear microscopy only clinics with no chest radiography. Potential gains need to be weighed against the likely increase in inappropriate 'false-positive' treatment. However, further impact-orientated studies focused on mortality and morbidity benefits in ill hospitalised patients are warranted.

\section{Additional file}

Additional file 1: Figure S1A. Pre-January 2014 LAM strip test manufacturer's reference card illustrating visual intensity grades 0-5. Figure S1B: January 2014 new LAM strip test manufacturer's reference card illustrating visual intensity grades $0-4$. Table $\mathrm{S1}$. Variables used to calculate the TB score as defined by Wejse et al. (2008) ${ }^{1}$. Each patient was scored at baseline, 2 months and 6 months. Table S2. Comparative diagnostic accuracy of two LAM strip test grade cut-points (old reference card) in HIV-infected patients and stratified by CD4 cell count. Table S3. Additional diagnostic accuracy measures (Likelihood ratios and predictive values) of LAM (grade 2 cut-point), sputum Xpert MTB/RIF or smear microscopy alone or in combination for culture-confirmed versus culture-negative pulmonary tuberculosis amongst HIV-infected (and refused testing) patients stratified by CD4 cell count (TB prevalence $=31 \%$ ). Table S4. Diagnostic accuracy of LAM (grade 2 cut-point) alone or in combination with either Xpert MTB/RIF or smear microscopy for culturepositive and clinical versus culture-negative pulmonary tuberculosis amongst HIV-infected (and refused testing) patients and stratified by CD4 cell count. Table S5A. Sensitivity of different diagnostic tests alone and in combination in HIV-infected (and refused testing) patients with culture-positive tuberculosis, stratified by study site. Table S5B. Specificity of different diagnostic tests alone or in combination in all patients culture-negative for tuberculosis, stratified by study site. Table S5C. Specificity of different diagnostic tests alone or in combination in HIV-infected patients culture-negative for tuberculosis and without clinical TB stratified by study site. Table S6. Changes in 2-month and 6-month TB-related morbidity indices in patients treated for TB according to baseline culture status, stratified by LAM result.

\section{Abbreviations}

LAM: Lipoarabinomannan. In manuscript used as abbreviation for Determine $^{\text {TM }}$ TB LAM Ag lateral flow strip test (Alere, USA); HIV: Human immunodeficiency virus; TB: Tuberculosis; EPTB: Extra-pulmonary tuberculosis; Xpert MTB/RIF: GeneXpert MTB/RIF assay (Cepheid, USA); POC: Point-of-care; DOTS: Directly observed Treatment Short-course; WHO: World Health Organisation; RCT: Randomised controlled trial; MGIT: Microscopic growth in-tube; $\mathrm{NaOH}$ : Sodium hydroxide; ZN: Ziehl-Neelsen; CD4: CD4 T-cell count; LR+: Positive likelihood ratio; PPV: Positive predictive value; LR-: Negative likelihood ratio; NPV: Negative predictive value; ELISA: Enzyme linked immunosorbent assay.

\section{Competing interests}

Although Alere supplied the ELISA and LAM strip-tests free of charge they had no role in the design and conduct of the study, analysis of the data or writing of the manuscript. The authors have no other interests to declare.

\section{Authors' contribution}

Conception and design: JP, GT, DC, PC, AR, MH, PMw, AP, KD. Study implementation: GT, JP, KD, AP, DC, PMw, AR, PC, MH. Analysis: GT, JP, DC, $M L, K D$. Interpretation and important intellectual input: GT, JP, DC, KD. First draft: JP, GT, DC, KD. All authors read and approved the final manuscript.

\section{Acknowledgments}

We are indebted to the patients who participated in this study. We thank the Health Directorate of the City of Cape Town, the Zambian Ministry of Health, the Kwa-Zulu Natal Provincial Department of Health, and the Tanzanian Ministry of Health and Social Welfare. We acknowledge the assistance of health facility staff at each site, and the assistance of the local institutional review boards.

\section{The TB-NEAT study team}

Field team: M Pretorius, M Pascoe, B Soetwater, M Wyngard, L Pool (Cape Town); C Viny, M Kasonde, L Manjeta (Lusaka); T Mthiyane, N Ntshuba, S Gumede, T Mvuyane, P Mbambo (Durban); C Mangu, F Kayombo, A Temihanga, M Kimaro, B Mnyanyi, I Mgogo, B Ambukege, T Sanga (Mbeya). Laboratory team: R Meldau, V Woodbourne, P Hope (Cape Town); J Mzyece (Lusaka); F Madaar (Durban); F Kayombo, H Mbilinyi, G. Rojas-Ponce, D Mapamba, C Lueer, A Bauer, L Njovu (Mbeya). Data team: D Cogill, V Louw (Cape Town); V Kapotwe (Lusaka); C Connolly (Durban); D Kowuor, N Chiwerengo, S Edwards (Mbeya).

\section{Author details}

${ }^{1}$ Lung Infection and Immunity Unit, Division of Pulmonology \& UCT Lung Institute, Department of Medicine, University of Cape Town, Cape Town, 
South Africa. ${ }^{2}$ TB Vaccine group, Jenner Institute, University of Oxford, Oxford, UK. ${ }^{3}$ Institute for Medical Research \& Training (IMReT), University Teaching Hospital, Lusaka, Zambia. ${ }^{4}$ National Institute of Medical Research, Mbeya Medical Research Centre, Mbeya, Tanzania. ${ }^{5}$ Division of Infectious Diseases and Tropical Medicine, Medical Centre of the University of Munich (LMU), Munich, Germany. ${ }^{6}$ German Centre for Infection Research (DZIF), Munich, Germany. ${ }^{7}$ Department of Medicine, University of Cape Town, Cape Town, South Africa. ${ }^{8}$ South African Medical Research Council, Durban, South Africa, KwaZulu Research Institute for Tuberculosis and HIV (K-RITH), Durban, South Africa. ${ }^{9}$ Institute of Infectious Diseases and Molecular Medicine, University of Cape Town, Cape Town, South Africa.

Received: 27 November 2014 Accepted: 29 May 2015 Published online: 09 July 2015

\section{References}

1. WHO. WHO Report 2013: Global tuberculosis control. Geneva: World Heath Organisation; 2013. ISBN 9789241564380.

2. Dowdy DW, Chaisson RE, Moulton LH, Dorman SE. The potential impact of enhanced diagnostic techniques for tuberculosis driven by HIV: a mathematical model. AIDS. 2006;20(5):751-62.

3. Lin HH, Dowdy D, Dye C, Murray M, Cohen T. The impact of new tuberculosis diagnostics on transmission: why context matters. Bull World Health Organ. 2012;90(10):739-47A. doi:10.2471/blt.11.101436. Epub 2012/10/31

4. Yoon C, Cattamanchi A, Davis JL, Worodria W, den Boon S, Kalema N, et al. Impact of Xpert MTB/RIF testing on tuberculosis management and outcomes in hospitalized patients in Uganda. PLoS One. 2012;7(11), e48599. doi:10.1371/journal.pone.0048599.

5. Theron G, Zijenah L, Chanda D, Clowes P, Rachow A, Lesosky M, et al. Feasibility, accuracy, and clinical effect of point-of-care Xpert MTB/RIF testing for tuberculosis in primary-care settings in Africa: a multicentre, randomised, controlled trial. Lancet. 2014;383(9915):424-35. doi:10.1016/ S0140-6736(13)62073-5.

6. Hanrahan CF, Selibas K, Deery CB, Dansey H, Clouse K, Bassett J, et al. Time to treatment and patient outcomes among TB suspects screened by a single point-of-care xpert MTB/RIF at a primary care clinic in Johannesburg. South Africa PLoS One. 2013;8(6), e65421. doi:10.1371/journal.pone.0065421.

7. Boehme CC, Nicol MP, Nabeta P, Michael JS, Gotuzzo E, Tahirli R, et al. Feasibility, diagnostic accuracy, and effectiveness of decentralised use of the Xpert MTB/RIF test for diagnosis of tuberculosis and multidrug resistance: a multicentre implementation study. Lancet. 2011;377(9776):1495-505. Epub 2011/04/22. doi:10.1016/S0140-6736(11)60438-8.

8. Theron G, Peter J, Dheda K. Xpert MTB/RIF test for tuberculosis. Lancet. 2011:378(9790):481. doi:10.1016/S0140-6736(11)61242-7. author reply 2-3. Epub 2011/08/09.

9. Peter JG, Theron G, Singh N, Singh A, Dheda K. Sputum induction to aid diagnosis of smear-negative or sputum-scarce tuberculosis in adults in HIV-endemic settings. Eur Respir J. 2014;43(1):185-94. doi:10.1183/ 09031936.00198012.

10. Alere. Alere Determine TB LAM Ag 2014 [cited 2014 7th April 2014]. Available from: http://www.alere.com/ww/en/product-details/determine-tblam.html.

11. Lawn SD, Kerkhoff AD, Vogt M, Wood R. Diagnostic accuracy of a low-cost, urine antigen, point-of-care screening assay for HIV-associated pulmonary tuberculosis before antiretroviral therapy: a descriptive study. Lancet Infect Dis. 2012;12(3):201-9. doi:10.1016/S1473-3099(11)70251-1.

12. Drain PK, Losina E, Coleman SM, Giddy J, Ross D, Katz JN, et al. Diagnostic accuracy of a point-of-care urine test for tuberculosis screening among newly-diagnosed hiv-infected adults: a prospective, clinic-based study. BMC Infect Dis. 2014;14(1):110. doi:10.1186/14712334-14-110. Epub 2014/02/28.

13. Balcha TT, Winqvist N, Sturegard E, Skogmar S, Reepalu A, Jemal ZH, et al. Detection of lipoarabinomannan in urine for identification of active tuberculosis among HIV-positive adults in Ethiopian health centres. Tropical medicine \& international health : TM \& H. 2014. doi:10.1111/ tmi.12308.

14. Nakiyingi L, Moodley VM, Manabe YC, Nicol MP, Holshouser M, Armstrong DT, et al. Diagnostic accuracy of a rapid urine lipoarabinomannan test for tuberculosis in HIV-infected adults. J Acquir Immune Defic Syndr. 2014. Epub 2014/03/29. doi:10.1097/qai.0000000000000151.
15. Lawn SD, Dheda K, Kerkhoff AD, Peter JG, Dorman S, Boehme CC, et al. Determine TB-LAM lateral flow urine antigen assay for HIV-associated tuberculosis: recommendations on the design and reporting of clinical studies. BMC Infect Dis. 2013;13:407. doi:10.1186/1471-2334-13-407.

16. Peter JG, Theron G, van Zyl-Smit R, Haripersad A, Mottay L, Kraus S, et al. Diagnostic accuracy of a urine lipoarabinomannan strip-test for TB detection in HIV-infected hospitalised patients. Eur Respir J. 2012;40(5):1211-20. doi:10.1183/09031936.00201711. Epub 2012/03/01.

17. Shah M, Ssengooba W, Armstrong D, Nakiyingi L, Holshouser M, Ellner JJ, et al. Comparative performance of urinary lipoarabinomannan assays and Xpert MTB/RIF in HIV-infected individuals with suspected tuberculosis in Uganda. AIDS. 2014. Epub 2014/03/19. doi:10.1097/qad.0000000000000264.

18. Drain PK, Grobler A, Gounder L, Sahid F, Wilson D, Bassett IV, et al. Rapid urine lipoarabinomannan testing after two months of tuberculosis treatment independently predicts mortality in a resource-limited setting. 44th Union World Conference on Lung Health; December, 2013. Paris: The International Journal of Tuberculosis and Lung Disease; 2013. p. S264.

19. Drain PK, Grobler A, Gounder L, Sahid F, Wilson D, Bassett IV, et al. Pointof-care urine lipoarabinomannan for diagnosis and treatment response of pulmonary tuberculosis in sputum smear-negative suspects. Int J Tuberc Lung Dis. 2013;17(12):S277.

20. Aderaye G. Improving the diagnosis and treatment of smear-negative pulmonary and extrapulmonary tuberculosis among adults and adolescents: recommendations for HIV-prevalent and resource-constrained settings: World Health Organization; 2007.

21. Getahun H, Harrington M, O'Brien R, Nunn P. Diagnosis of smearnegative pulmonary tuberculosis in people with HIV infection or AIDS in resource-constrained settings: informing urgent policy changes. Lancet. 2007;369:2042-9.

22. Wejse C, Gustafson P, Nielsen J, Gomes VF, Aaby P, Andersen PL, et al. TBscore: Signs and symptoms from tuberculosis patients in a low-resource setting have predictive value and may be used to assess clinical course. Scand J Infect Dis. 2008;40(2):111-20. doi:10.1080/00365540701558698.

23. Dean AG SK, Soe MM. OpenEpi: Open Source Epidemiologic Statistics for Public Health, Version 2.3.1. http://www.OpenEpi.com, updated 2010/19/09, accessed 2010/11/30.

24. Development Core Team. R: A language and environment for statistical computing. Vienna, Austria: R Foundation for Statistical Computing; 2005. ISBN 3-900051-07-0.

25. Bossuyt PM, Reitsma JB, Bruns DE, Gatsonis CA, Glasziou PP, Irwig LM, et al. The STARD statement for reporting studies of diagnostic accuracy: explanation and elaboration. Ann Intern Med. 2003;138(1):W1-12. Epub 2003/01/07. doi:200301070-00012-W1.

26. Peter JG, Theron G, Dheda K. Urine antigen test for diagnosis of HIV-associated tuberculosis. Lancet Infect Dis. 2012;12(11):825. doi:10.1016/s14733099(12)70220-7. author reply 6-7. Epub 2012/10/27.

27. Shah M, Martinson NA, Chaisson RE, Martin DJ, Variava E, Dorman SE. Quantitative analysis of a urine-based assay for detection of lipoarabinomannan in patients with tuberculosis. J Clin Microbiol. 2010;48(8):2972-4. doi:10.1128/ jcm.00363-10. Epub 2010/06/11.

28. Talbot E, Munseri P, Teixeira P, Matee M, Bakari M, Lahey T, et al. Test characteristics of urinary lipoarabinomannan and predictors of mortality among hospitalized HIV-infected tuberculosis suspects in Tanzania. PLoS One. 2012;7(3):e32876. doi:10.1371/journal.pone.0032876. Epub 2012/03/14.

29. Gounder CR, Kufa T, Wada NI, Mngomezulu V, Charalambous S, Hanifa Y, et al. Diagnostic Accuracy of a Urine Lipoarabinomannan Enzyme-Linked Immunosorbent Assay for Screening Ambulatory HIV-Infected Persons for Tuberculosis. J Acquir Immune Defic Syndr. 2011;58(2):219-23. doi:10.1097/ QAl.0b013e31822b75d4. Epub 2011/07/19.

30. Dheda K, Davids V, Lenders L, Roberts T, Meldau R, Ling D, et al. Clinical utility of a commercial LAM-ELISA assay for TB diagnosis in HIV-infected patients using urine and sputum samples. PLoS One. 2010;5(3), e9848. doi:10.1371/journal.pone.0009848. Epub 2010/03/31.

31. Mutetwa R, Boehme C, Dimairo M, Bandason T, Munyati SS, Mangwanya D, et al. Diagnostic accuracy of commercial urinary lipoarabinomannan detection in African tuberculosis suspects and patients. Int J Tuberc Lung Dis. 2009;13(10):1253-9. Epub 2009/10/02.

32. Daley P, Michael JS, Hmar P, Latha A, Chordia P, Mathai D, et al. Blinded evaluation of commercial urinary lipoarabinomannan for active tuberculosis: a pilot study. Int J Tuberc Lung Dis. 2009;13(8):989-95. Epub 2009/09/03. 
33. Reither K, Saathoff E, Jung J, Minja LT, Kroidl I, Saad E, et al. Low sensitivity of a urine LAM-ELISA in the diagnosis of pulmonary tuberculosis. BMC Infect Dis. 2009;9:141.

34. Lawn SD, Edwards DJ, Kranzer K, Vogt M, Bekker LG, Wood R. Urine lipoarabinomannan assay for tuberculosis screening before antiretroviral therapy diagnostic yield and association with immune reconstitution disease. AIDS. 2009;23(14):1875-80. Epub 2010/01/29.

35. Drain PK, Losina E, Coleman SM, Giddy J, Ross D, Katz JN, et al. Value of urine lipoarabinomannan grade and second test for optimizing clinic-based screening for HIV-associated pulmonary tuberculosis. J Acquir Immune Defic Syndr. 2015:68(3):274-80. doi:10.1097/QAl.0000000000000436.

36. Theron G, Peter J, van Zyl-Smit R, Mishra H, Streicher E, Murray S, et al. Evaluation of the Xpert MTB/RIF assay for the diagnosis of pulmonary tuberculosis in a high HIV prevalence setting. Am J Respir Crit Care Med. 2011;184(1):132-40. doi:10.1164/rccm.201101-0056OC.

37. Steingart KR, Schiller I, Horne DJ, Pai M, Boehme CC, Dendukuri N. Xpert(R) MTB/RIF assay for pulmonary tuberculosis and rifampicin resistance in adults. The Cochrane Database Syst Rev. 2014;1:CD009593. doi:10.1002/ 14651858.CD009593.pub3.

38. Treatment Action Group MSF. Paris meeting on TB point-of-care test specifications. 2009.

39. Kroidl I, Clowes P, Mwakyelu J, Maboko L, Kiangi A, Rachow A, et al. Reasons for false-positive lipoarabinomannan ELISA results in a Tanzanian population. Scand J Infect Dis. 2014:46(2):144-8. doi:10.3109/00365548.2013.853133. Epub 2013/11/28

40. Peter JG, Theron G, Dheda K. Can point-of-care urine LAM strip testing for tuberculosis add value to clinical decision making in hospitalised HIV-infected persons? PLoS One. 2013;8(2):e54875. doi:10.1371/journal.pone.0054875. Epub 2013/02/08

41. Peter JG, Theron G, Muchinga TE, Govender U, Dheda K. The diagnostic accuracy of urine-based Xpert MTB/RIF in HIV-infected hospitalized patients who are smear-negative or sputum scarce. PLoS One. 2012;7(7):e39966. doi:10.1371/journal.pone.0039966.

42. Minion J, Leung E, Talbot E, Dheda K, Pai M, Menzies D. Diagnosing tuberculosis with urine lipoarabinomannan: systematic review and meta-analysis. Eur Respir J. 2011;38(6):1398-405. doi:10.1183/09031936.00025711. Epub 2011/06/28.

\section{Submit your next manuscript to BioMed Central and take full advantage of:}

- Convenient online submission

- Thorough peer review

- No space constraints or color figure charges

- Immediate publication on acceptance

- Inclusion in PubMed, CAS, Scopus and Google Scholar

- Research which is freely available for redistribution 